\title{
Doubling spectroscopy challenge
}

\author{
Reinhard Meusinger ${ }^{1}$
}

(C) Springer-Verlag Berlin Heidelberg 2015

We would like to invite you to participate in the Analytical Challenge, a series of puzzles to entertain and challenge our readers. This special feature of "Analytical and Bioanalytical Chemistry" has established itself as a truly unique quiz series, with a new scientific puzzle published every other month. Readers can access the complete collection of published problems with their solutions on the "Analytical and Bioanalytical Chemistry" homepage at http://www.springer. com/abc. Test your knowledge and tease your wits in diverse areas of analytical and bioanalytical chemistry by viewing this collection.

In the present challenge, doubling phenomena is the topic. And please note that there is a prize to be won (a Springer book of your choice up to a value of $€ 100)$. Please read on...

\section{Meet the doubling spectroscopy challenge}

Today we will not deal with the structure determination of a biochemical compound as is typical for this challenge. Rather, this challenge will start with the given structure of the compound: $\beta$-D-2',3'-didehydro-2',3'-dideoxy-5-fluorocytidine (D-D4FC), shown in Fig. 1. Afterwards we will discover two doubling phenomena in the proton nuclear magnetic resonance spectrum of this cytosine nucleoside.

Reinhard Meusinger

meusi@oc.chemie.tu-darmstadt.de

1 Institute of Organic Chemistry and Biochemistry, University of Technology Darmstadt, Alarich-Weiss-Str. 4,

64287 Darmstadt, Germany

\section{The challenge}

The eventful history of this compound started as a potent antiviral agent for human immunodeficiency virus (HIV). It was designated DPC 817 by DuPont Company laboratories. DPC 817 was licensed by DuPont from Pharmasset, a biotech firm founded in Atlanta (US) by the notable anti-HIV drug creator Raymond Schinazi. In spring of 2001, DuPont pharmaceutical unit, which had at this time few promising AIDS drugs in development, was acquired by the Bristol-Myers Squibb (BMS) company. Shortly after the acquisition, BMS decided to return DPC 817 to Pharmasset Ltd., where it was dubbed as Reverset ${ }^{\mathrm{TM}}$. In 2003, the pharmaceutical company Incyte revealed that Incyte and Pharmasset had entered into a collaborative licensing agreement to develop and commercialize the anti-retroviral drug Reverset. Two years later, the US Food and Drug Administration (FDA) indicated that the proposed brand name Reverset would not be acceptable and the generic name dexelvucitabine (DFC) was to be used. Finally, on 3 April 2006, Incyte announced launching further trials and the development of the experimental nucleoside reverse transcriptase inhibitor DFC. The decision was based on several cases of increased amylase levels (hyperlipasemia), a sign of

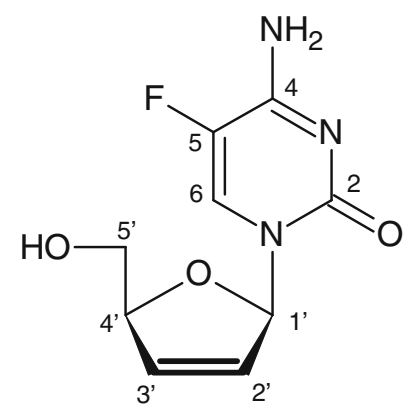

Fig. 1 Formula 1 
Fig. $2500 \mathrm{MHz}{ }^{1} \mathrm{H}-\mathrm{NMR}$ of DFC in DMSO-D ${ }_{6}$. (Courtesy of Dr. Vibhuti Klingler-Dabral. Bio De Tek, Griesheim, Germany)

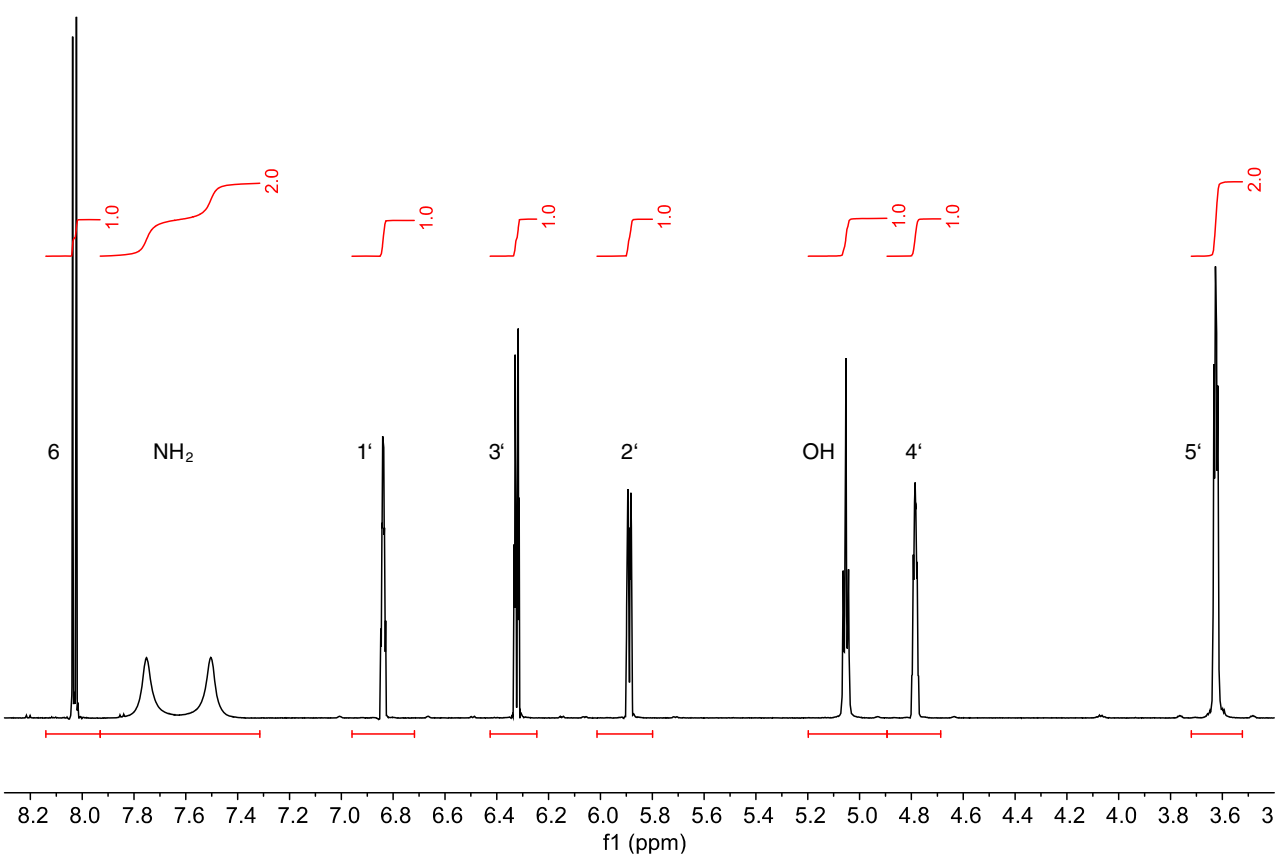

pancreas inflammation. At this time, the experimental agent was already being studied in a Phase II clinical trial as a "oncedaily" anti-HIV drug.

In Fig. 2, the ${ }^{1} \mathrm{H}-\mathrm{NMR}$ spectrum of DFC is shown. It looks exactly as described in the first paper, was found for this substance, published in 1999 [1].
Owing to the fact that the chemical shift difference of the diasterotopic methylene protons in $5^{\prime}$ position is too small, only nine signals were observed here for the 10 hydrogen atoms. Their assignment is given in Fig. 2. Let us look more closely at the downfield shifted signals assigned to H-6 and $\mathrm{NH}_{2}$ protons. Here, the special feature is the splitting of both
Fig. 3 Region from $7.2 \mathrm{ppm}$ to $8.2 \mathrm{ppm}$ of the $300 \mathrm{MHz}{ }^{1} \mathrm{H}-$ NMR spectrum (top) and $500 \mathrm{MHz}$ spectrum (above) of DFC, drawn at the same scale ratios of ppm/cm (left) and $\mathrm{Hz} / \mathrm{cm}$ (right), respectively

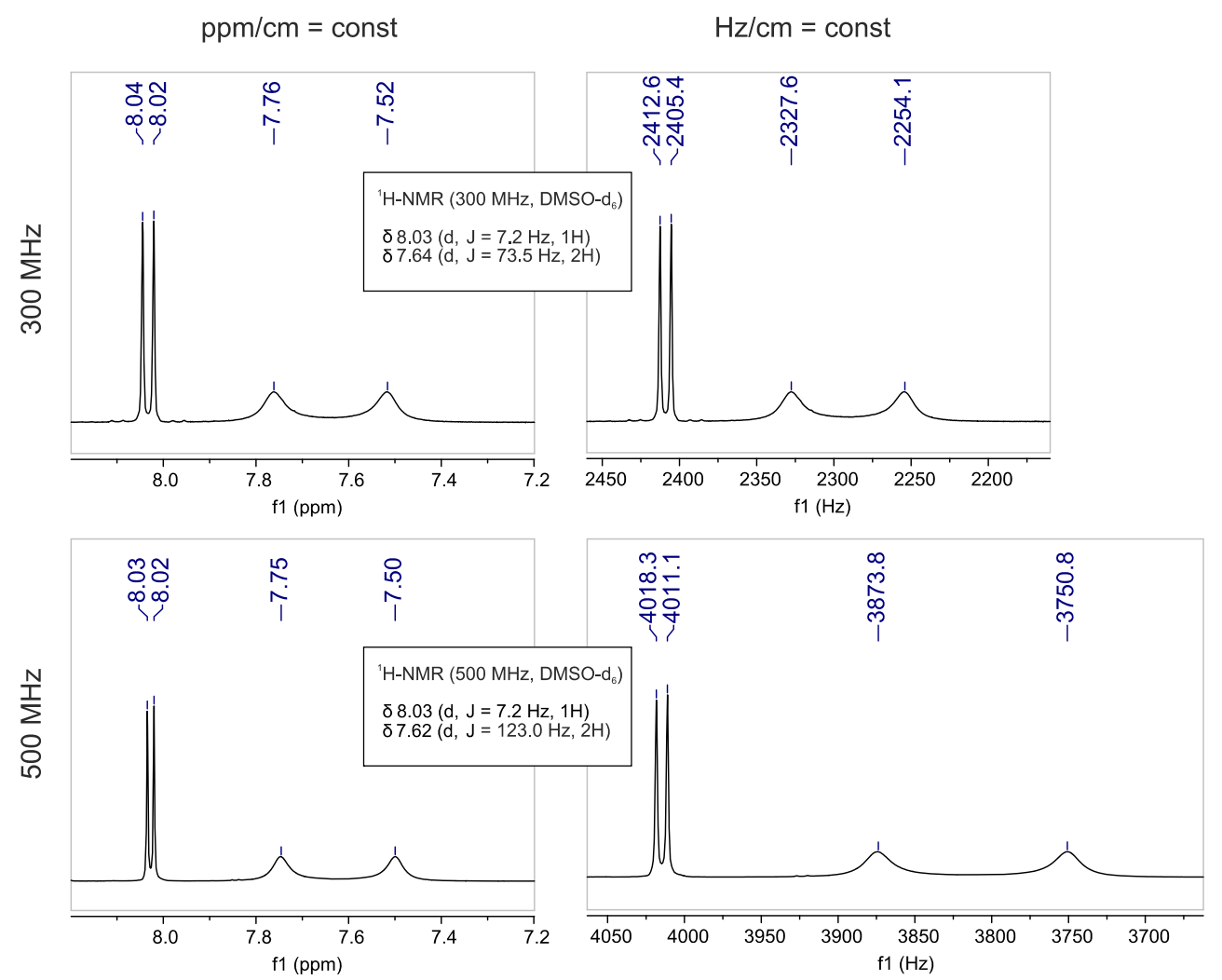


signals into two lines each. While the signal of the H-6 proton looks like a common doublet, the protons of the amino group deliver two broad lines with a marked difference in their chemical shifts. This is shown more detailed in Fig. 3.

Here, the same range of the ${ }^{1} \mathrm{H}-\mathrm{NMR}$ spectrum is pictured four times. The two upper spectra were measured at the $300 \mathrm{MHz}$ excitation frequency, whereas the two lower spectra were measured at $500 \mathrm{MHz}$. In this way, it became apparent that spacing between the H-6 lines is independent of the field strength when it is measured in $\mathrm{Hz}(7.2 \mathrm{~Hz})$ in contrast to the $\mathrm{NH}_{2}$ signals $(73.5 \mathrm{~Hz}$ and $123 \mathrm{~Hz}$, respectively). So, the H-6 signal is indeed a doublet and the separation of the two lines is a coupling constant $J_{6, X}$. Usually, the coupling observed in a ${ }^{1} \mathrm{H}-$ NMR spectrum is the result of the interaction between nearby protons. This phenomenon is described comprehensibly for the two aromatic protons in cytosine by Clayden, Greeves, and Warren in their text book "Organic chemistry" [2]. But different from the compound cytosine, the position 5 is not occupied by a coupling-causing proton in DFC, the compound discussed in this challenge. A long range coupling to one of the other hydrogen atoms will be excluded by the ${ }^{1} \mathrm{H},{ }^{1} \mathrm{H}$-COSY spectrum shown in Fig. 4, unambiguously. No cross peak appendant to the $\mathrm{H}-6$ doublet is detected here.

So, the first question is: What is the reason for the splitting of the H-6 signal in ${ }^{1} H-N M R$ spectrum of DFC?

The second question results from the remarkable doubling of the amino group signal. Usually, amino groups show only a<smiles>[R]n1cc(F)c(NP)nc1=O</smiles>

Fig. 5 Formula 2

single ${ }^{1} \mathrm{H}-\mathrm{NMR}$ signal, often broadened for different reasons. As a matter of fact, amino group signals are observable only in aprotic solvents like DMSO. As shown in Fig. 3, the shift difference of the two observed lines depends on the magnetic field strength. Evidently, the doubling is not caused by a spin-spin coupling. Here, the two amino protons are in different chemical environments and have different resonance frequencies (Fig. 5).

Because of the high energy barrier to internal rotation around the $\mathrm{C} 4-\mathrm{NH}_{2}$ bond, the residence time of the two $\mathrm{NH}_{2}$ hydrogen atoms in positions near F-5 or near N-3 is relatively long. Consequently, two separate signals are observed at room temperature. If the temperature is raised, these signals broaden until they coalescence into a single line. This phenomenon, known as chemical exchange or dynamic NMR, is shown in Fig. 6.

The goal of this experiment is to derive a relationship between the rate constant and the temperature. By measuring the
Fig. 4 The two-dimensional ${ }^{1} \mathrm{H}$ correlated double quantum filtered COSY spectrum of DFC

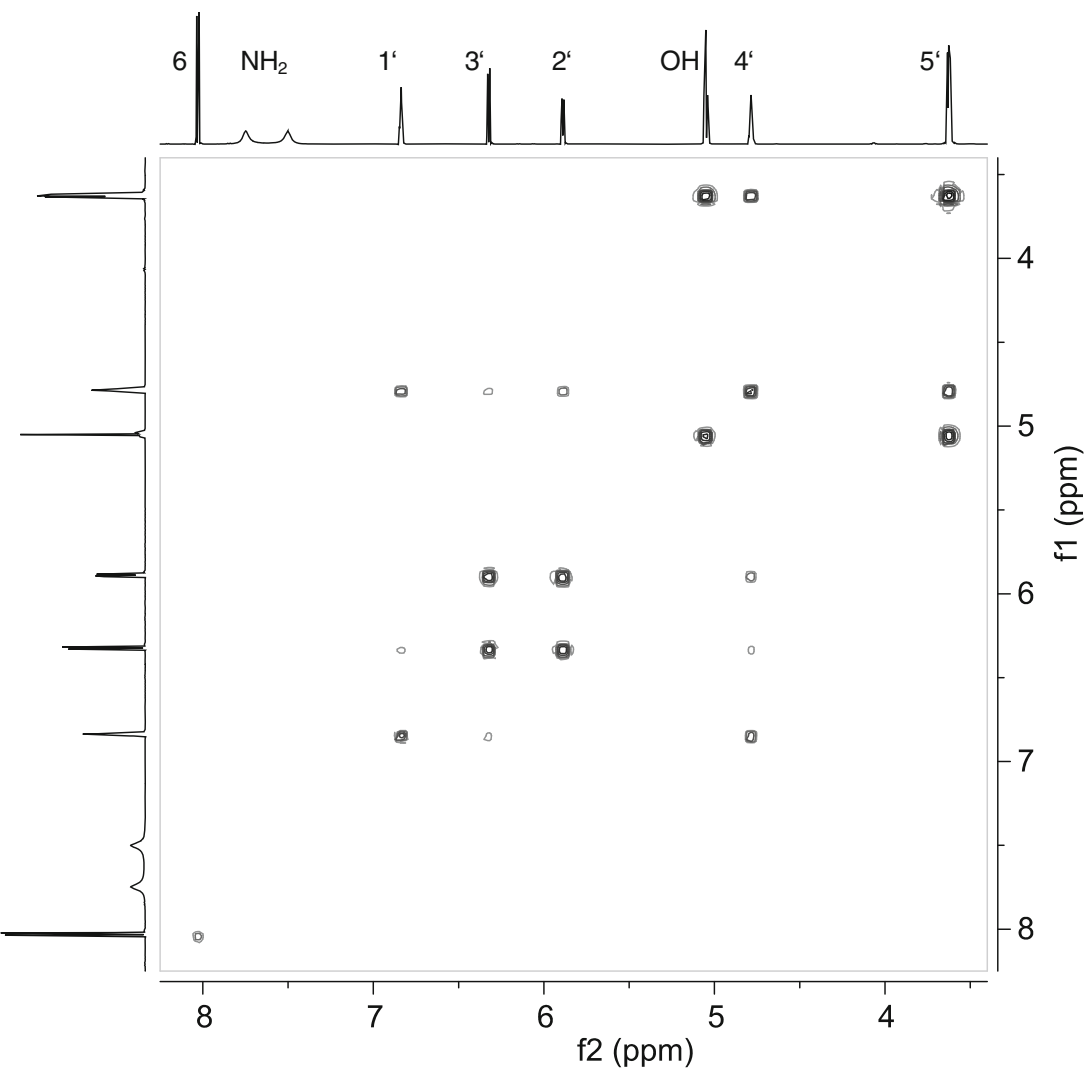


Fig. 6 Part of the temperature depended $500 \mathrm{MHz}$ (left) and $300 \mathrm{MHz}$ (right) ${ }^{1} \mathrm{H}-\mathrm{NMR}$ spectra of DFC in DMSO-D . The exact temperatures are shown beside the spectra. The spectra at the coalescence temperature $(*)$ are marked by a thick line

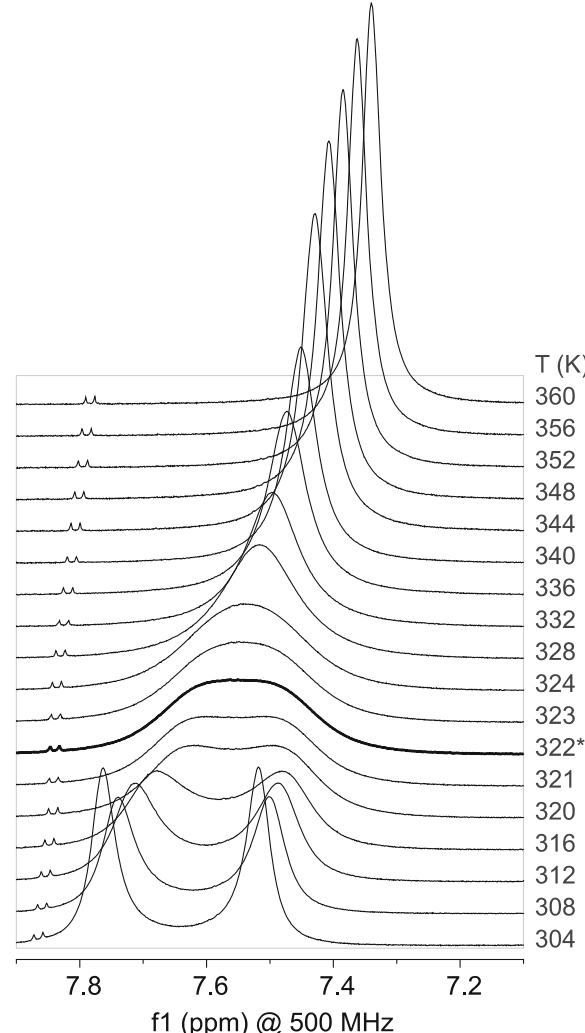

temperature and by using the Eyring equation, one can derive the Gibbs energy of activation $\left(\Delta G^{\#}\right)$, the enthalpy of activation $\left(\Delta H^{\#}\right)$, and the entropy of activation $\left(\Delta S^{\#}\right)$ for the observed process.

Here, starting at room temperature, a slow exchange is observed between rotamers. Their separated signals merge into a broad band at the coalescence point $\left({ }^{*}\right)$ and become a singlet in the region of fast exchange by further heating. This enables one to use the Eyring equation to make a quick evaluation of the energy barrier for the exchange process of the amino protons at the coalescence temperature $T_{\mathrm{c}}$ :

$k_{\text {rate }}=\frac{k_{\mathrm{B}} T}{h} e^{-\frac{\Delta G^{\#}}{R T}}$

where $k_{\mathrm{B}}$ is the Boltzmann constant, $h$ is the Planck constant, and $R$ is the universal gas constant. In addition, the rate constant, $k_{\text {rate }}$, at this temperature is given as $k_{\text {rate }}=\pi \Delta v / \sqrt{2}$ where $\Delta v$ is the frequency difference between involved protons (see Fig. 3). Combining these expressions leads to Gibbs energy of the activation:

$$
\begin{aligned}
\Delta G^{\#} & =R T_{\mathrm{c}} \ln \frac{\sqrt{2} k_{\mathrm{B}} T_{\mathrm{c}}}{\pi h \Delta v} \\
& =R T_{\mathrm{c}}\left(\ln \frac{\sqrt{2} k_{\mathrm{B}}}{\pi h}+\ln \left(T_{\mathrm{c}} / \Delta v\right)\right) .
\end{aligned}
$$

Obviously, the coalescence temperature, $T_{\mathrm{c}}$, and the frequency difference, $\Delta \nu$, both depend on the magnetic field strength.

The second question is: What is the energy barrier for the exchange process of the amino protons in $D F C$ ?

\section{References}

1. Shi J, Jeffrey McAtee J, Schlueter Wirtz S, Tharnish P, Juodawlkis A, Liotta DC, Schinazi RF (1999) J Med Chem 42:859-867

2. Clayden J, Greeves N, Warren S (2012) Organic chemistry. Oxford University Press, Oxford, p 285

We invite our readers to participate in the Analytical Challenge by solving the puzzle above. Please send the correct solution to abc-challenge@springer.com by 1 December 2015. Make sure you enter "Doubling spectroscopy challenge" in the subject line of your e-mail. The winner will be notified by e-mail and his/her name will be published on the "Analytical and Bioanalytical Chemistry" homepage at $\mathrm{http}: / / \mathrm{www}$.springer.com/abc and in the journal (volume 408/issue 7) where the readers will find the solution and a short explanation.

The next Analytical Challenge will be published in Issue 408/1, January 2016. If you have enjoyed solving this Analytical Challenge, you are invited to try the previous puzzles on the $A B C$ homepage. 